\title{
Klippel-Trenaunay-Weber Syndrome - A case report and review of the literature
}

\author{
Florentina Anca Danciu, Catalina Bistriceanu*, Lucian Cracana
}

"Prof. Dr. N. Oblu" Neurosurgery Clinical Emergency Hospital, lasi, Romania

\begin{abstract}
Klippel-Trenaunay-Weber syndrome (KTW syndrome), also known as congenital dysplastic angiopathy or angioosteohypertrophy syndrome, is a rare, sporadic, complex malformation that involves congenital malformations of veins, capillaries, and/or lymphatics, which leads to soft tissue hypertrophy and port-wine stains. We report a case of a 37 year old woman with congenital Klippel Trenaunay Weber syndrome. She had many vascular abnormalities from birth which increased in time such that she had many surgical interventions. These abnormalities of veins and capillaries were leading to hypertrophy of the extremities - finger macrosomia. She had orthopedic comorbidities like Chopart amputation of the anterior part of her left foot and dorsal kyphoscoliosis because of the external scars. Studying similar cases from the literature, we found that Klippel-Trenaunay-Weber syndrome could be associated with brain abnormalities. We admitted the patient for additional investigations because some of these patients could have cerebral malformations in the absence of neurologic abnormalities. We considered at that time that it was necessary to look for any unknown vascular abnormality. Management of this syndrome should aim to correct any of the abnormalities present, if technically possible, and if the abnormality is causing symptoms.
\end{abstract}

Keywords: Klippel-Trenaunay-Weber syndrome, port-wine stain, varicose veins, arterio-venous communication.

\section{Introduction}

Klippel-Trenaunay-Weber syndrome (KTWS) is characterized by a triad of port-wine stain, varicose veins, and bone and soft tissue hypertrophy involving an extremity [1].

This syndrome presents at birth or during early infancy or childhood [2]. The etiology of the Klippel-Trenaunay-Weber syndrome remains obscure.

Some authors suggest that KTW syndrome is caused by mesodermal abnormality during fetal development, leading to the maintenance of microscopic arteriovenous communications in the limb bud,

Received: October 2014; Accepted after review: November 2014; Published: December 2014

${ }^{*}$ Corresponding author: Catalina Bistriceanu, MD, Neurology Department, "Prof. Dr. N. Oblu" Neurosurgery Clinical Emergency Hospital, 1 Ateneului Str., lasi, Romania. Email: catalina nastac@yahoo.com as a result of which the triad of nevus, hypertrophy, and superficial varices is produced [3]. We are presenting a case of uncomplicated KTW syndrome and a brief review of the literature.

\section{Case report}

A 37 year old woman known with congenital Klippel-Trenaunay-Weber syndrome is admitted into our clinic with vertigo and mild pulsatile headache localized „in vertex”. She has no family history of congenital malformations. At age 24, her family doctor guided her to the Surgical Department for appropriate treatment. During the intervention the diagnosis was established and this was later confirmed by histopathological exam. 
Personal history: Her vascular abnormalities were present from birth and gradually increased in size.

Over time, there were numerous surgical interventions towards the removal of the parietal hemangiomas.

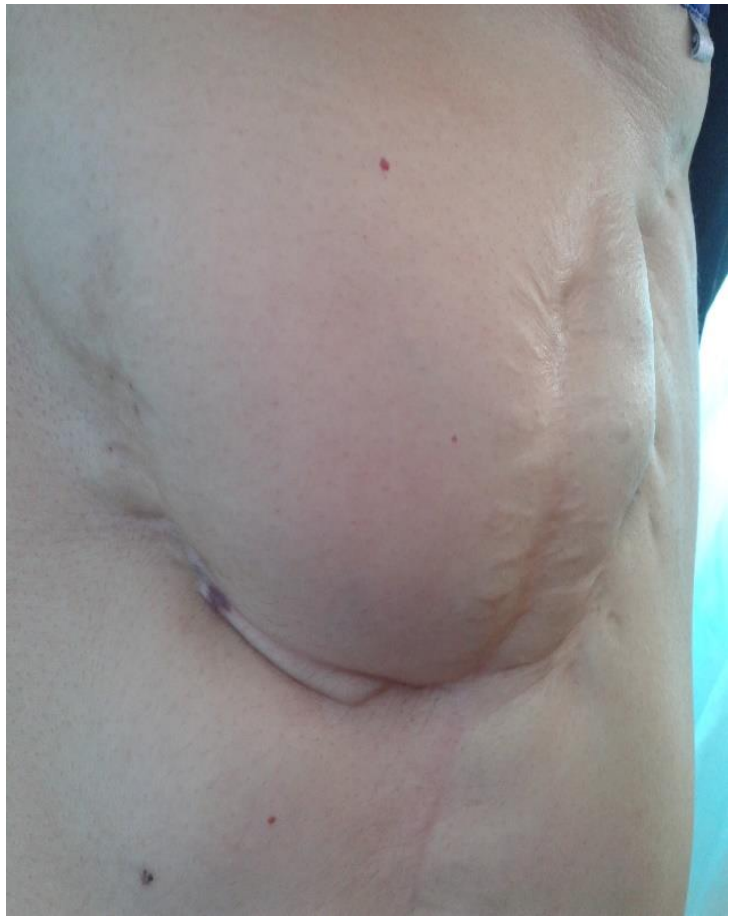

Fig. 1. The scar of a surgically removed parietal hemangioma and the remained vascular malformation

On physical examination we observed: multiple postoperative scars on her abdomenright arm, left hypochondrium, left hemithorax; vascular tumor in left hypochondrium (Figure 1); finger macrosomia with congenital hypertrophy of right hand third finger (Figure 2 and Figure 3); hypertrophy of left hand second-third finger (Figure 4); partial left foot amputation; dorsal hyphoscoliosis (external cause - scars).

The neurologic examination revealed trophic disorders due to vascular abnormalities (asymmetrical aspect of the upper limbs) (Figure 5), left Claude Bernard Horner syndrome: left eye ptosis (grade I-II), left eye miosis (Figure 6), without any other pathological signs.

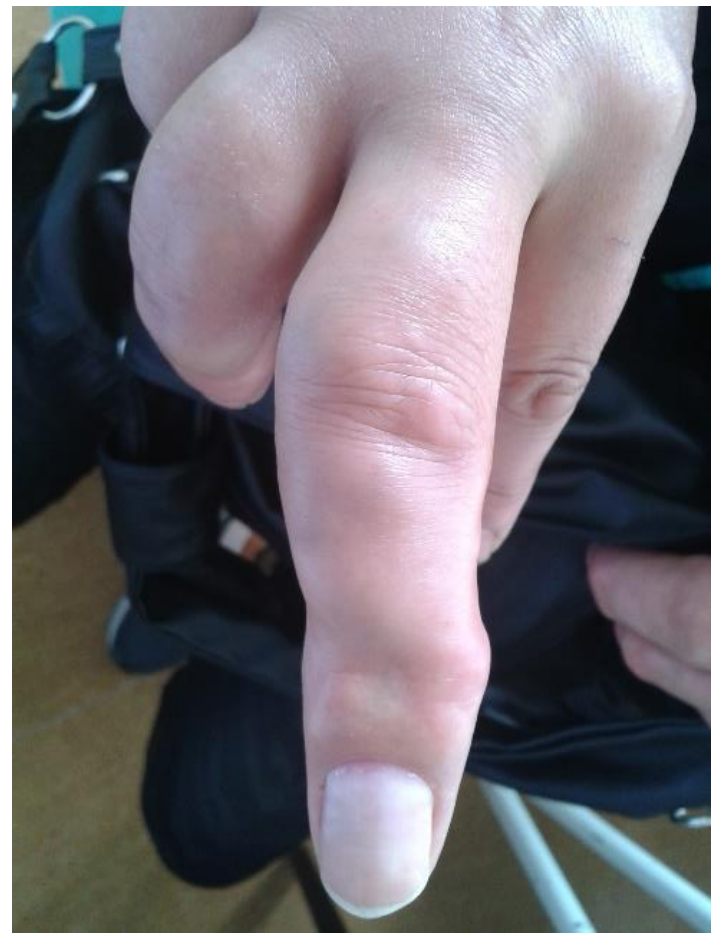

Fig. 2. Hypertrophy of right hand third finger

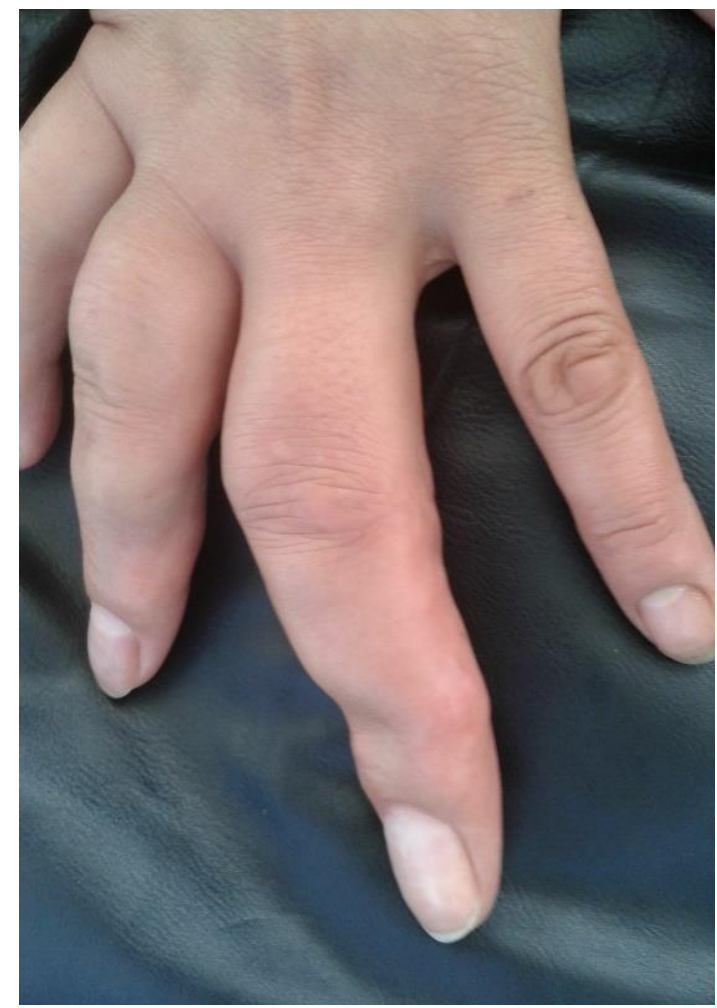

Fig. 3. Hypertrophy of right hand third finger 


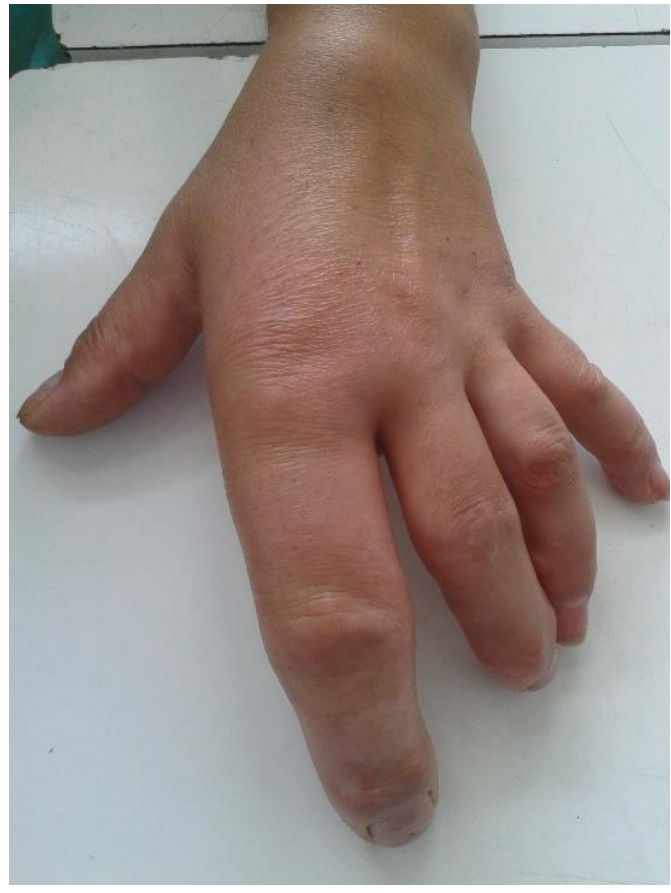

Fig. 4. Hypertrophy of left hand second-third finger

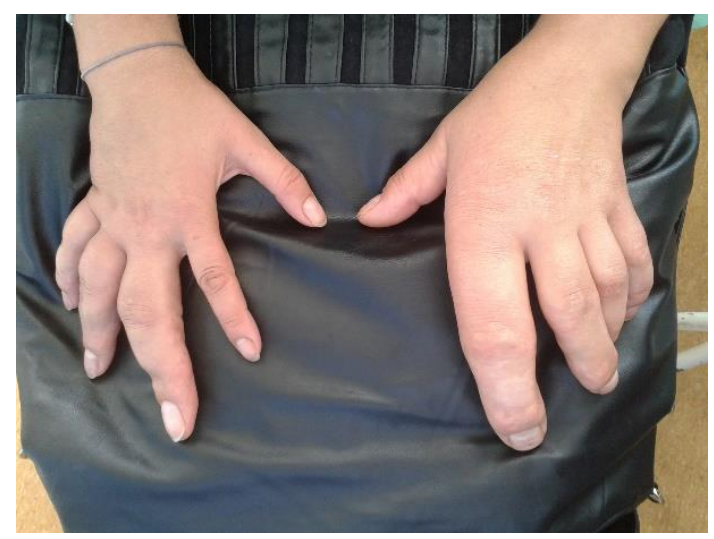

Fig. 5. Distal trophic disorders secondary to vascular ingrowth

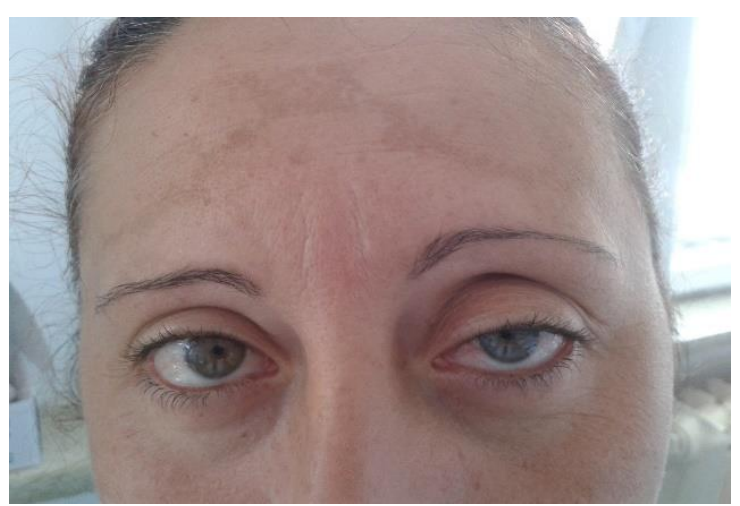

Fig. 6. Left Claude Bernard Horner syndrome

Other investigations. Eye examination: left eye ptosis degree I-II; exophtalmometria: normal; intraocular pressure: right $=15 \mathrm{mmHg}$; left $=14 \mathrm{mmHg}$; iris heterochromia; left eye: pinguecula. Psychological exam: recurrent depressive reaction (mourning). Pulmonary Xray: mediastinal enlargement. Cervical Xray: cervical spine rectitude; reduced C5-C6 space. EKG: sinusal rhythm, $60 \mathrm{BPT}$, inverted T wave in V2-V4, flattened $\mathrm{T}$ wave in D3, AVF, and V6. Electroencephalography: increased theta rhythm predominantly in right cerebral hemisphere and rare delta multiforme waves. Peripheral blood analysis: normal. Cerebral CT: normal.

\section{Discussions}

Brain abnormalities of Klippel-TrenaunayWeber syndrome include hemorrhage, infarction, hydrocephalus, cerebral or cerebellar hemihypertrophy, cerebral calcifications, vascular malformation, cavernoma, arteriovenous malformations, aneurysm, choroid plexus abnormalities, cortical dysplasia, leptomeningeal enhacement $[4,5]$.

In our case we didn't find any cerebral modifications. We consider that her left Claude Bernard Horner syndrome is secondary to the upper thoracic interventions with the injury of cervical plexus.

\section{Brief review of the literature}

The association of varicose veins, soft tissue and bony hypertrophy and „port-wine” variety of cutaneous hemangiomas confined to one extremity was first reported in 1900 by Klippel and Trenauney. In 1907, ParkesWeber described a second entity which appears to be similar to the Klippel-Trenauney triad with the addition of an arteriovenous fistula [6]. The etiology of this syndrome remains obscure although some hypotheses are postulated. Recent studies establish VG5Q (angiogenic factor) as a susceptibility gene for KTW syndrome and show that increased angiogenesis is a molecular pathogenic mechanism of KTW syndrome [7]. Various cases of this syndrome are presented in literature. Despite this, incidence and 
genetic predisposition of this rare disease has not been established. Clinical presentation of patients with KTW syndrome has a wide spectrum from incomplete, mild forms of portwine stains and few varicose veins causing only cosmetic deformity, to severe disability associated with massive limb overgrowths, chronic pain syndrome, skin infections, arthritis, thromboembolism and life-threatening or recurrent bleeding from venous malformations.

Table 1. Major findings that led to the diagnosis of KTW syndrome

\begin{tabular}{l}
\hline Cutaneous hemangioma - port wine stain \\
\hline Long-standing varicose veins \\
Soft tissue and bony hypertrophy of the extremities \\
Arterio-venous malformation
\end{tabular}

Diagnosis of KTW syndrome is mainly clinical (Table 1). Investigations of this syndrome should be focused on evaluation of the type, extent and severity of the malformation, and on confirming the absence of any clinically significant arteriovenous shunting [8].

Management is largely conservative and the extent of diagnostic evaluation is determined by the planned treatment. A multidisciplinary approach to management of KTW syndrome is warranted [9]. There is no cure for this disorder. Therapeutic objectives seek to improve the patient's condition and treat the consequences of severe lesions. Treatment of port-wine stains is usually done with pulsed dye laser therapy that yields better results when applied to lesions in the face and trunk, as compared to extremities [10]. Nevertheless, it only contributes to the superficial treatment of hemangiomas. When varicose veins are present, compression stockings are recommended for venous insufficiency. Surgical treatment is only recommended in symptomatic cases of superficial varicose veins. The use of orthopedic braces is a good option to prevent the development of vertebral deformities in case of hypertrophy of the lower limbs. With time, corrective bone surgery may be necessary to treat significant limb length discrepancy, if present [11].

The lower limb is the site of malformation in approximately $95 \%$ of patients. When found on the trunk, the malformation rarely crosses the midline. The hypertrophy involves the length as well as the circumference of the involved extremity and is caused by local hyperemia and venous stasis secondary to the vascular anomaly [12].

\section{Conclusions}

The management of patients with KTW syndrome continues to be primarily nonsurgical, but those patients with patent deep veins can be considered for excision of symptomatic varicose veins. Although the recurrence rate is high, clinical improvement is significant and reoperations can be performed if needed.

Because KTW syndrome is rare, patients should receive multidisciplinary care in qualified vascular centers [13]. We conclude that the management of KTW syndrome includes careful diagnosis, prevention and treatment of complications. 
Authors declare no conflicts of interest.

\section{References}

1. Sadiq MF, Shuaib W, Tiwana MH, Johnson JO, Khosa F. Klippel-Trénaunay syndrome with intracranial arteriovenous malformation: a rare presentation. Case Rep Radiol. 2014; 2014, doi:10.1155/2014/202160.

2. Kihiczak GG, Meine JG, Schwartz RA, Janniger CK. Klippel-Trenaunay syndrome: a multisystem disorder possibly resulting from a pathogenic gene for vascular and tissue overgrowth. Int J Dermatol 2006; 45(8):88390.

3. Baskerville PA, Ackroyd JS, Browse NL. The etiology of the Klippel-Trenaunay syndrome. Ann Surg 1985; 202(5):624-7.

4. Torregrosa A, Marti-Bonmati L, Higueras V, Poyatos C, Sanchis A. Klippel-Trenaunay syndrome: frequency of cerebral and cerebellar hemihypertrophy on MRI. Neuroradiology 2000; 42(6):420-3.

5. Renard D, Larue A, Taieb G, Jeanjean L, Labauge $P$. Recurrent cerebral infarction in Klippel-Trenaunay-Weber syndrome. Clin Neurol Neurosurg 2012; 114(7):1019-20. doi: 10.1016/j.clineuro.2012.01.024.

6. Lindenauer SM. The Klippel-Trenaunay Syndrome: varicosity, hypertrophy and hemangioma with no arteriovenous fistula Ann Surg 1965; 162(2):303-314.

7. Tian X-L, Kadaba R, You S-A, et al. Identification of an angiogenic factor that when mutated causes susceptibility to KlippelTrenaunay syndrome. Nature 2004; 427:640645.

8. Zea MI, Hanif M, Habib M, Ansari A. KlippelTrenaunay Syndrome: a case report with brief review of literature. J Dermatol Case Rep 2009; 3(4):56-59.

9. Gloviczki P, Driscoll DJ. Klippel-Trenaunay syndrome: current management. Phlebology 2007; 22(6):291-8.

10. Jacob AG, Driscoll DJ, Shaughnessy WJ, Stanson AW, Clay RP, Gloviczki P. KlippelTrénaunay syndrome: Spectrum and management. Mayo Clin Proc 1998; 73(1):2836.

11. Karim T, Singh $U$, Nanda NS. A rare presentation of Klippel Treanunay syndromeIndian Dermatol Online J 2014; 5(2):1546. doi: 10.4103/2229-5178.131086.

12. Hale EK. Klippel-Trenaunay syndrome. Dermatol Online J 2002; 8(2):13.

13. Noel AA, Gloviczki P, Cherry KJ Jr, Rooke TW, Stanson AW, Driscoll DJ. Surgical treatment of venous malformations in KlippelTrénaunay syndrome. J Vasc Surg 2000; 32(5):840-7. 